\title{
The Effect Observation of Lianhua Qingwen Granule in the Adjuvant Treatment of Community Acquired Pneumonia (CAP)
}

\author{
Xia Jianzhen $^{1}$, Shi Tongxia ${ }^{1}$, Cao Wenli ${ }^{2}$ * \\ ${ }^{1}$ Outpatient 1st Department, Community Hospital of Minzu University of China, Beijing, China \\ ${ }^{2}$ Infection 1st Department, Beijing Geriatric Hospital, Beijing, China
}

Email address:

1211123766@qq.com (Xia Jianzhen),13161220446@163.com (Cao Wenli)

${ }^{*}$ Corresponding author

\section{To cite this article:}

Xia Jianzhen, Shi Tongxia, Cao Wenli. The Effect Observation of Lianhua Qingwen Granule in the Adjuvant Treatment of Community Acquired Pneumonia (CAP). Science Discovery. Vol. 9, No. 4, 2021, pp. 155-159. doi: 10.11648/j.sd.20210904.15

Received: April 12, 2021; Accepted: June 2, 2021; Published: June 4, 2021

\begin{abstract}
Objective To observe the efficacy of Lianhua Qingwen Granule in the adjuvant treatment of community-acquired pneumonia (CAP). Methods From March 2018 to January 2020, adult patients with CAP who visited the internal medicine clinic of community health service center of Minzu University of China were divided by a random code list into two groups: the observation group (31 cases), Lianhua Qingwen Granule orally combined with Levofloxacin intravenous drop, The control group (31 cases), Levofloxacin intravenous drop alonely. The cough symptom score, the cough visual simulation score (VAS), antipyretic time and X-ray chest shadow absorption were compared between the two groups to judge the curative effect. Measurement data were expressed as mean \pm standard deviation, and comparison used independent sample $t$ test. Enumeration data were expressed as percentages, total effective rates were compared by $\chi^{2}$ tests. Results There were no statistically significant differences in baseline data, such as age, score of cough symptom before treatment between the two groups. Compared with the control group, cough symptom score and VAS of observation group decreased significantly $[(2.45 \pm 1.312)$ point vs $(3.61 \pm 1.476)$ point, $\mathrm{P}=0.002],[(3.68 \pm 1.973)$ point vs $(4.84 \pm 1.753)$ point, $\mathrm{P}=0.017]$, antipyretic time was decreased significantly [(3.00 $\pm 1.4814)$ day VS $(4.842 \pm 1.5728)$ day, $\mathrm{P}=0.001]$, effective rate was increased significantly $(87.1 \% \mathrm{VS} 64.5 \%, \mathrm{P}=0.038)$. Compliance: the rate of hospital transfer without medical advice was decreased significantly $(6.45 \% \mathrm{VS} 29.03 \% \mathrm{P}=0.046)$. Conclusion Lianhua Qingwen Granule combined with levofloxacin has good efficacy and compliance in the treatment of CAP, and community doctors can choose it.
\end{abstract}

Keywords: Community Acquired Pneumonia (CAP), Lianhua Qingwen Granule, Levofloxacin Injection, The Community Hospital, Compliance

\section{连花清瘟颗粒辅助治疗社区获得性肺炎疗效观察}

\author{
夏建珍 ${ }^{1}$, 史同霞 ${ }^{1}$, 曹文利 ${ }^{2 *}$ \\ ${ }^{1}$ 中央民族大学中央民族大学社区服务中心门诊一部, 北京, 中国 \\ ${ }^{2}$ 北京老年医院感染一科, 北京, 中国 \\ 邮箱 \\ 1211123766@qq.com（夏建珍），13161220446@163.com（曹文利）
}

摘要：目的：观察连花清瘟颗粒辅助左氧氟沙星治疗社区获得性肺炎（CAP）的疗效。方法：收集2018年3月至2020 年1月门诊就诊的符合CAP的患者, 按随机对照原则分为观察组（31例）：连花清瘟颗粒口服联合左氧氟沙星静滴; 对 
照组（31例）：单用左氧氟沙星静滴。比较两组患者治疗前、后咳嗽症状积分、咳嗽视觉模拟评分（VAS）、退热时 间及X线阴影吸收情况。计量资料采用均数土标准差表示, 比较采用独立样本 $\mathrm{t}$ 检验。计数资料用百分比表示, 比较采 用 $\chi^{2}$ 检验。结果 两组患者年龄、治疗前咳嗽症状积分等基线资料比较, 差异无统计学意义。与对照组比较: 观察组咳 嗽症状积分及VAS明显下降, 分别为 [ (2.45 \pm 1.312$)$ 分 VS(3.61 \pm 1.476$)$ 分, $\mathrm{P}=0.002]$ [(3.68 \pm 1.973$)$ 分vs (4.84 \pm 1.753$)$ 分, $\mathrm{P}=0.017]$; 退热时间明显减少, 为(3.00 \pm 1.4814$)$ 天VS(4.842 \pm 1.5728$)$ 天, $\mathrm{P}=0.001$; 有效率明显增加, 为 $87.1 \% \mathrm{VS} 64.5 \%$, $\mathrm{P}=0.038$; 依从性: 非医嘱转院率显著减少, 为 $6.45 \% \mathrm{VS} 29.03 \%, \mathrm{P}=0.046$ 。结论 连花清瘟颗粒联合左氧氟沙星治疗 $\mathrm{CAP}$ 的疗效好, 依从性好, 是社区医生治疗 CAP的可靠方案。

关键词：社区获得性肺炎, 连花清瘟颗粒, 左氧氟沙星注射液, 社区医院, 患者依从性

\section{1. 引言}

社区获得性肺炎(CAP)是指在医院外易患的感染性 肺实质 (含肺泡壁即广义上的肺间质) 炎症, 包括具有明 确潜伏期的病原体感染而在入院后潜伏期内发病的肺炎 [1]。CAP 作为临床较常见的感染性疾病, 在健康系统的 产生了重大经济负担 [2]。大多数CAP患者是轻症, 可以在 门诊接受治疗, 社区医院可以提供门诊服务, 因此社区医 生在CAP 的管理策略和治疗方案上可发挥重要决策作用 [3]。社区医生经验性采用左氧氟沙星等喹诺酮类抗生素治 疗门诊CAP取得一定的疗效[4-5]。但仍有一些患者因体温 持续升高, 咳嗽症状不减轻等原因, 对社区医院的诊疗技 术及治疗疗效存在质疑, 涌向人满为患的大医院, 既增加 了经济负担, 占用了宝贵的医疗资源, 又增加了医院内感 染的风险。如何提高患者在社区医院初始治疗的疗效及依 从性, 中西医结合的方法或能解决一部分问题。连花清瘟 作为中成药在抗病毒、退热等方面有较强的功效, 在上呼 吸道感染等疾病中有较广泛的应用 [6]。但检索文献发现连 花清瘟在下呼吸道尤其是CAP的应用研究资料尚不多见。 本研究主要探讨连花清瘟颗粒联合左氧氟沙星注射液治 疗CAP的疗效及患者的依从性。

\section{2. 方法}

\section{1. 研究对象}

收集2018年3月-2020年1月中央民族大学社区卫生服 务中心内科门诊就诊的符合 CAP的成人患者 62 例, 年龄 18-76岁, 其中男性患者29例, 女性患者33例, 就诊前未曾 服用抗生素治疗。CAP患者诊断符合中华医学会呼吸病学 分会制定的《社区获得性肺炎诊断和治疗指南》的诊断标 准[1]。排除标准:（1）存在肺部基础疾病（如慢性阻塞性 肺疾病、肺肿瘤、肺结核、肺水肿、肺不张、支气管扩张 等);（2）存在严重肾脏、肝脏病变、急性心脑血管病及
免疫功能缺陷等严重疾病者; (3) 门诊就诊时评估 CRB-65[7](意识障碍; 呼吸频率 $\geq 30$ 次 $/ \mathrm{min}$; 低血压（收 缩压 $<90 \mathrm{~mm} \mathrm{Hg}$ ( $1 \mathrm{~mm} \mathrm{Hg}=0.133 \mathrm{kPa}$ )或舒张压 $<60 \mathrm{~mm} \mathrm{Hg}$ ) 及年龄 $\geq 65$ 岁。以上四项每一项（有）计算 1 分，（无）计 算0分)积分 $\geq 2$ 分; (4) 在社区医院治疗不足 3 天或不能配 合观察疗效的患者。所有患者既往无喹诺酮类应用禁忌, 治疗前行胸片, 血常规, 血压, 肺部听诊检查。按照随机 数字编码表分为治疗组和对照组。观察组 31 例 (男8例, 女 23例）, 发热19例, 咳嗽29例, 胸痛2例, 合并症: 高血压 6例, 糖尿病2例。对照组31例（男10例, 女21例）, 发热 19例, 咳嗽30例, 胸痛2例, 合并症: 高血压2例, 糖尿病4 例。两组患者一般资料比较差异无统计学意义 $(\mathrm{P}>0.05)$ 。

\section{2. 治疗方法}

观察组: 连花清瘟颗粒 (生产厂家: 北京以岭药业有 限公司, 生产批号2004016）1代tid口服联合左氧氟沙星注 射液静滴（生产厂家：扬子江药业集团有限公司，生产批 号19082652）200ml/0.4qd; 对照组单用左氧氟沙星注射液 静滴 $200 \mathrm{ml} / 0.4 \mathrm{qd}$ 。两组均给予其它综合性治疗, 包括化痰、 解痉止咳及支持治疗, 治疗期间体温 $\geq 38.5^{\circ} \mathrm{C}$ 给予物理降 温或解热镇痛药物, 不使用其他抗生素、糖皮质激素及抗 病毒药物。两组患者原则上治疗7天, 7 天后观察疗效, 根 据《社区获得性肺炎诊断和治疗指南》推荐, 在用药后的 48至72小时内对初始治疗药物的应用进行评价. 如果患者 出现持续高热, 咳嗽加重, 甚至意识障碍等临床情况, 及 时由医生转院至医联体合作三级医院, 以免贻误病情。

\section{3. 观察指标}

\subsection{1. 咳嗽积分 (Cough Score)}

咳嗽症状积分表分为日间积分和夜间积分, 两部分均 按照不同的轻重程度分为 $0 、 1 、 2 、 3$, 这四个等级 (表 1 ), 咳嗽症状积分为日间积分+夜间积分, 所有患者均记录治 疗前及治疗 3 天后咳嗽症状积分 $[8]$ 。

表1 咳嗽积分表。

\begin{tabular}{lll}
\hline 分值 & 日间咳嗽积分 & 夜间咳嗽积分 \\
\hline 0分 & 无咳嗽 & 无咳嗽 \\
1 分 & 偶有短暂咳嗽 & 入睡时短暂咳嗽 \\
2 分 & 频繁咳嗽, 轻度影响日间生活 & 因咳嗽, 轻度影响夜间睡眠 \\
3 分 & 频繁咳嗽, 严重影响生活 & 因咳嗽, 严重影响睡眠 \\
\hline
\end{tabular}




\subsection{2. 视觉模拟评分体系（VAS）}

采用线性计分法, 作一刻度为0-10刻度的直线, 0 刻 度表示无咳嗽, 10 刻度表示最严重程度的咳嗽, 由患者主 观对自己咳嗽程度进行评分, 数值越大, 表示患者咳嗽程 度越严重。所有患者均记录治疗前及治疗 3 天后视觉模拟 评分体系评分数值 $[8]$ 。

\subsection{3. 记录患者每天体温}

退热时间 (体温降至正常后24小时不再发热, 定为退 热，体温高于 $37.3^{\circ} \mathrm{C}$ 持续的时间为退热时间）。

\subsection{4. $\mathrm{X}$ 线胸片检查}

初次就诊时检查胸片, 如果患者症状恶化明显, 48-72 小时内可以复查胸片, 其他患者由于影像滞后于临床的情 况, 治疗 1 周后复查 $[9]$ 。

\subsection{5. 其他症状}

如胸痛症状, 意识状态, 血压及呼吸频率等临床情况。 记录两组治疗期间并发症发生情况，包括肠道不适、 穿刺血管刺激征等。

\section{4. 疗效评价}

\subsection{1. 显效}

体温正常, 咳嗽、胸痛等临床症状消失, 胸部X线提 示炎性反应完全吸收;

\subsection{2. 有效}

体温下降, 咳嗽、咯痰、胸痛明显减轻, 胸部 $\mathrm{X}$ 线示 炎性反应有所吸收;

\subsection{3. 无效}

体温仍高, 咳嗽、咯痰、胸痛无明显减少, 胸片无明 显改善。治疗中途（初始治疗48-72小时对疗效进行评估 时) 因疾病进展需要转至二、三级医院治疗者, 视为无效。 治疗期间, 患者因自觉症状缓解不明显等因素, 在社区医 院就诊的依从性差, 要求转院治疗, 亦定为治疗无效。

治疗 3 天后与治疗前比较, 咳嗽症状积分下降 3 分及以 上或视觉评分下降3分及以上为显效, 下降1-2分为有效, 无下降或上升者视为无效。

[ (显效+有效 $) /$ 该组病例总数 $] \times 100 \%$ 记为总有效率。

\section{5. 统计学分析}

应用SPSS17.0统计软件包进行统计学分析, 计量资料 采用均数土标准差表示, 符合正态性、方差齐性时采用独 立样本 $\mathrm{t}$ 检验, 如不符合正态分布, 则行Wilcoxon 秩和检验。 计数资料以百分比表示, 比较采用 $\chi^{2}$ 检验。 $\mathrm{P}<0.05$ 为差异 有统计学意义。

\section{3. 结果}

\section{1. 两组基线资料及治疗前后指标比较}

本研究共收集62例患者, 两组患者年龄, 性别, 治疗 前咳嗽积分, VAS评分等基线资料比较差异无统计学意义 $(\mathrm{P}>0.05$, 表 2$)$ 。治疗 3 天后, 患者咳嗽积分、VAS评分明 显下降, 治疗7天后, 患者退热时间缩短, 两组患者比较 差异有统计学意义 $(* \mathrm{P}<0.05$, 表 2$)$ 。

表2 两组患者基线资料及治疗前、治疗后 3 天咳嗽症状积分、视觉模拟评分及退热时间比较 $(\bar{x} \pm s)$ 。

\begin{tabular}{llllllll}
\hline 组别 & 性别 (男/女) & 年龄 (岁) & $\begin{array}{l}\text { 治疗前咳嗽 } \\
\text { 积分 (分) }\end{array}$ & $\begin{array}{l}\text { 治疗前VAS评 } \\
\text { 分 (分) }\end{array}$ & $\begin{array}{l}\text { 治疗3 天后咳 } \\
\text { 嗽积分 (分) }\end{array}$ & $\begin{array}{l}\text { 治疗3 天后 } \\
\text { VAS评分 (分) }\end{array}$ & $\begin{array}{c}\text { 退热时间 } \\
\text { (天) }\end{array}$ \\
\hline 观察组 & $8 / 23$ & $46.10 \pm 16.013$ & $3.39 \pm 1.202$ & $4.74 \pm 1.861$ & $2.45 \pm 1.312$ & $3.68 \pm 1.973$ & $3.000 \pm 1.481$ \\
对照组 & $10 / 21$ & $46.42 \pm 17.002$ & $3.77 \pm 0.884$ & $4.84 \pm 1.241$ & $3.61 \pm 1.476$ & $4.84 \pm 1.753$ & $4.842 \pm 1.572$ \\
$\chi^{2} / t$ 值 & 0.313 & 0.340 & 3.267 & 0.952 & 1.247 & 0.453 & 2.133 \\
$P$ 值 & 0.576 & 0.939 & 0.234 & 0.154 & $0.002^{*}$ & $0.017^{*}$ & $0.001^{*}$ \\
\hline
\end{tabular}

\section{2. 两组治疗后疗效及依从性比较}

观察组 31 例中 4 例治疗 7 天后复查胸片, 阴影完全吸收, 判断为显效。23例发热、咳嗽症状或胸片复查减轻, 判断 为治疗有效。4例判断为治疗无效者分别: 1 例因持续咳嗽, 高热于治疗 72 小时后, 医嘱转院治疗; 1 例治疗 7 天后, 复 查胸片, 肺部阴影无减轻或出现新的阴影, 医嘱建议转院 进一步诊治; 2例因症状自觉好转不明显, 治疗 3 天后非医 嘱自行转至其他医院治疗。对照组 31 例中 2 例治疗 7 天后复 查胸片, 阴影完全吸收, 判断为显效。18例治疗7天后症 状减轻, 复查胸片, 阴影减轻, 判断为治疗有效。11例判 断为无效分别是：1例持续高热、咳嗽, 72 小时复查胸片 病情进展, 医嘱转院; 1 例治疗 7 天后复查胸片阴影减轻不
明显, 医嘱转上级医院进一步诊治; 9例治疗 3 天后自觉仍 有咳嗽、发热, 非医嘱自行转其他医院治疗。7天后疗效 比较, 观察组总有效率 $87.1 \%$, 对照组 $64.5 \%$, 差异有统计 学意义 $(* 1 \mathrm{p}<0.05$ 见表 3$)$, 治疗过程中两组非医嘱转院 率观察组 $6.5 \%$, 对照组 $29.0 \%$, 两组患者依从性存在差异, 差异有统计学意义 $(* 2 \mathrm{p}<0.05$ 见表 3$)$ 。

\section{3. 两组不良反应情况}

两组治疗过程中左氧氟沙星注射液出现轻度血管刺 激反应 3 例, 经调慢输液速度, 自行好转, 未影响治疗。 治疗组有 2 例口服连花清瘟颗粒有轻微腹泻, 能耐受, 未 影响治疗。 
表3 两组患者治疗7天后疗效比较[例（\%）]。

\begin{tabular}{lllllll}
\hline 组别 & 例数 & 无效 & 显效 & 有效 & 总有效 & 非医嘱转院 \\
\hline 观察组 & 31 & $4(12.9)$ & $4(12.9)$ & $23(74.2)$ & $27(87.1)$ & $2(6.5)$ \\
对照组 & 31 & $11(35.5)$ & $2(6.5)$ & $18(58.1)$ & $20(64.5)$ & $9(29.0)$ \\
$\chi 2$ 2值 & & & & & 4.309 & 3.979 \\
$\mathrm{P}$ 值 & & & & & $0.038^{* 1}$ & $0.046^{* 2}$ \\
\hline
\end{tabular}

\section{4. 讨论}

国内一项成人CAP流行病学调查 [10]结果显示: CAP 病原体主要是细菌, 其中肺炎链球菌是最多见的致病菌之 一，而非典型病原体及病毒的感染率也在逐年增加。美国 ATS指南[7]指出门诊CAP最常见病原体是肺炎链球菌、流 感嗜血杆菌、肺炎支原体、肺炎衣原体及呼吸道病毒等。 陈亮等[11]对3381例无吸入因素的CAP患者进行病原学分 析, 其中 365 例 (10.8\%) 获得病原学诊断, 肺炎链球菌和 甲型流感病毒是CAP最常见的病原体。体外药效学研究 [12]结果显示左氧氟沙星对肺炎链球菌、肺炎克雷伯菌、 流感嗜血杆菌等具有良好作用。王晓文[13]研究发现左氧 氟沙星针对老年患者肺炎支原体治疗有效率达 $76.92 \%$ 。综 上可见, 左氧氟沙星能覆盖 $\mathrm{CAP}$ 常见的致病细菌及支原体 等病原体。本研究中对照组单独使用左氧氟沙星治疗 CAP, $64.5 \%$ 患者胸片的肺部阴影情况及症状有减轻或完全消失, 临床有疗效。但是还有三分之一以上的患者临床疗效不佳, 这可能与左氧氟沙星不能覆盖的致病细菌、耐药支原体或 病毒感染有关。国内多项研究表明, 9.6-36.4\%的门诊CAP 患者存在病毒感染[14-15], Zhan等[16]研究指出, 呼吸道 病毒是肺炎常见的病因, 病毒性肺炎最常见的症状是发热、 疲劳。由此可见, 治疗CAP应该加用抗病毒药物辅助治疗, 但目前除了抗流感病毒应用奥司他韦[17]等神经氨酸酶抑 制剂外, 无其他特效抗病毒的西药, 社区医院因各种条件 限制较难获得奥司他韦等西药。而中成药连花清瘟有抗病 毒作用, 在国家基本药物目录, 社区药房常备药物, 比较 容易为患者开具。

连花清瘟是国家卫生部首推抗甲型流感病毒的一 线用药 [18-19]。连花清瘟的处方由连翅、金银花、多 麻黄、板蓝根、石膏、炒苦杏仁、绵马贯众、大黄、 鱼腥草、广雚香、薄荷脑、红景天及甘草组成。连疑、 金银花、板蓝根等 7 种药物对流感病毒的蛋白酶有抑制 作用; 石膏降低流感病毒引起的高热; 炎麻黄和苦杏 仁有止咳、平喘和兴奋中枢的药理作用; 红景天提高 人体的免疫力。丁月文等 $[20]$ 研究表明, 连花清瘟在体 外减轻病毒诱导的炎症因子基因表达, 抑制不同菌株 的流感病毒增殖, 对流感病毒具有广谱效应, 尤其对 病毒感染的免疫应答具有调节作用, 另外, 丁月文等 [21] 还在研究呼吸道病合胞病毒感染小鼠中发现连花 清瘟颗粒可抑制肺内合胞病毒滴度, 对病毒性肺炎肺 组织具有一定的改善修复作用。刘钊等 [21]研究发现连 花清瘟能有效地抑制柯萨奇病毒B4在 Hep-2 细胞增 殖, 抑制病毒进入细胞之后的复制增殖, 直接灭活病 毒, 并阻断病毒吸附正常功能的细胞。王妍等[23]研究 发现, 连花清瘟颗粒联合常规治疗儿童支原体肺炎临
床疗效明显, 可以调节机体免疫功能, 抑制炎症反应, 显著改善患儿肺功能。陈列强等 [24]研究发现: 连花清 瘟可降低血清炎症因子水平, 快速缓解支原体肺炎患 儿临床症状如（发热、肌肉酸痛）, 疗效显著。董樑 等 [25]研究发现连花清瘟可以通过抑制相关炎症因子 IL-8、TNF-a的释放, 从而显著改善慢性阻塞性肺疾病 急性加重期的症状, 增加临床治疗效果。本研究连花 清瘟颗粒联合左氧氟沙星注射液观察组总有效率 $87.10 \%$, 对照组为 $64.51 \%, \mathrm{P}<0.05$, 差异有显著性。 加用连花清瘟明显缩短了患者退热时间, 减轻咳嗽、 咳疾症状, 增加了患者在社区医院诊治的依从性, 对 照组非医嘱转院率为 $29.0 \%$, 而观察组为 $6.5 \%$, 两组差 异有显著性。陈波等[26]利用连花清瘟联合莫西沙星治 疗老年CAP, 总有效率达 $94.43 \%$, 而单用莫西沙星有 效率 $76.32 \%$ 。吕宏等 [27]连花清瘟联合左氧氟沙星口服 治疗肺炎 14 天, 治疗总有效率 $96.67 \%$, 较单用左氧氟 沙星具有较好的临床疗效, 并且治疗组患者肿瘤坏死 因子- $\alpha(\mathrm{TNF}-\alpha)$ 、超敏反应 C 蛋白 (hs-CRP) 和白 介素-4 (IL-4) 水平等炎性指标均较对照组显著降低。 本研究及以上多项研究表明观察组疗效确切, 充分表 明左氧氟沙星等呼吸喹诺酮类抗生素能覆盖大多数 CAP致病菌及支原体等病原体, 连花清瘟能抗病毒, 并且能辅助抗菌、抗支原体及抑制炎症因子的释放。

\section{5. 结论}

综上所述, 连花清瘟颗粒联合左氧氟沙星注射液治疗 $\mathrm{CAP}$, 中西药联合应用, 增加了社区医生治疗 CAP的疗效, 快速改善患者的症状, 增加了患者在社区医院治疗的依从 性。社区医生在制定 CAP诊疗方案过程中可以选择应用连 花清瘟颗粒和左氧氟沙星联合治疗的方案。

本研究选取的病例比较少, 与很多CAP患者首诊直 接去大医院有关, 未来将充分发挥社区医生在社区工作 的优势, 多向患者宣传疾病的预防、诊疗知识、相关的 医保政策及鼓励社区居民积极接种流感疫苗及肺炎疫 苗等, 履行初级卫生医疗保健工作者的责任, 增加患者 的认可度, 一旦出现疾病的症状首诊选择社区医院, 提 高患者疗效, 树立良好的口碑, 提高患者在社区就诊的 依从性。

\section{参考文献}

[1] 中华医学会呼吸病学分会. 中国成人社区获得性肺炎诊断 与治疗指南(2016版)[]].中华结核和呼吸杂志, 2016, 39(4): 253-279. 
[2] Broulette J, Yu H, Pyenson B, et al. The Incidence Rate and Economic Burden of Community-Acquired Pneumonia in a Working-Age Population[J]. Am Health Drug Benefits, 2013, 6(8):494-503.

[3] AKHTER S, RIZVI N, BHURA S, et al. Management of community acquired pneumonia by family physicians[J].Pak J Med Sci, 2017, 33 (4) : 783-787.

[4] 杨凯, 朱云颖, 肖婷婷, 等. 2019年美国IDSA/ATS成人社 区获得性肺炎诊疗指南更新特点 $[\mathrm{J}$ ] 。 中华临床感染病 杂志, 2019,12(5):339-343.

[5]陈宇红.我院成人社区获得性肺炎抗菌药物用药合理性分 析 [J].临床医药文献电子杂，2019，6（79）:10-11.

[6] 陈丽云, 王银娣, 黄汉. 连花清瘟胶囊抗流感病毒临床疗 效观察 $[\mathrm{J}] . 甘$ 甘肃医药, 2017.36（8）：666-667.

[7] Metlay JP, Waterer GW, Long AC, et al. Diagnosis and treatment of adults with community-acquired pneumonia: an official clinical practice guideline of the American Thoracic Society and Infectious Disease Society of America[J].AmJ Respir Crit Care Med, 2019,200（7）:e45-e67.

[8] 中华医学会呼吸病学分会哮喘学组. 咳嗽的诊断与治疗指 南(2015) [J].中华结核和呼吸杂志, 2016, 39(5): 323-354.

[9] 余业洲, 赵红, 邹立巍等. 社区获得性肺炎治疗前后的影 像学表现[J].中国医学影像学杂志, 2019, 27 (9): 674-676.

[10] 刘又宁，陈民钧，赵铁梅等．中国城市成人社区获得性肺 炎665例病原学多中心调查[J].中华结核和呼吸杂志, 2006, 29(1): 3-8.

[11] 陈亮, 韩秀迪, 李艳丽等. 具有吸入因素的社区获得性肺 炎住院患者临床特征分析 [J]. 医学研究生学报, 2020, 33 (1): 54-61.

[12] 张菁, 郁继诚, 施耀国等. 左氧氟沙星药代动力学/药效学研 究[J].中华医学杂志, 2005, 85（27）：1926-1932.

[13] 王晓文. 左氧氟沙星联合痰热清治疗老年支原体肺炎的临 床分析[J].中华实验和临床感染病杂志:电子版, 2015, 9(3): 387-389.

[14] Liu YF, Gao Y, Chen MF, et al. Etiological analysis and predictive diagnostic model building of community-acquired pneumonia in adult outpatients in Beijing, China[J].BMC Infect Dis, 2013, 13: 309.
[15] 胡月,王宇, 刘景院等.社区获得性肺炎病原学及耐药现状研 究进展 $[J]$. 中华实验和临床感染病杂志(电子版),2018,12(3).

[16] Zhan Y, Yang Z, Chen R, et al. Respiratory virus is a real pathogen in immunocompetent community-acquired pneumonia: comparing to influenza like illness and volunteer controls[J].BMC Pulm Med, 2014, 14: 144.

[17] 吕巍巍, 贾鹏, 张志贤等. 磷酸奥司他韦治疗流行性感冒 的临床疗效及不良反应分析 [J]. 黑龙江医学, 2018, 42(8): 808,810 .

[18] 丁新㑆. 连花清瘟药物抗流感病毒的药理分析[J].临床合理 用药，2012，5（10A）:32.

[19] 王守军.连花清瘟胶囊抗流感病毒药理机制研究[J].临床合 理用药杂志， 2019，12（05）：53-54.

[20] Ding YW, Zeng LJ, Li RF, et al. The Chinese prescription lianhuaqingwen capsule exerts anti-influenza activity through the inhibition of viral propagation and impacts immune function[J].BMC Complementary and Alternative Medicine 2017, 17 (1):130.

[21] 丁月文, 曾丽娟, 李润峰等. 连花清瘟颗粒抗呼吸道合胞 病毒感染BALB / c 小鼠的药效作用研究 $[\mathrm{J}]$. 广州中医药大 学学报, 2016, 33 (4):540-544.

[22] 刘钊, 石福忠, 杨占秋. 连花清瘟胶囊抗柯萨奇 B4 病毒 作用的实验研究 [J].中南民族大学学报(自然科学版), 2012, 31(1):20 - 24.

[23] 王妍, 彭经纬, 覃涛. 连花清瘟颗粒对儿童肺炎支原体肺炎 肺功能及炎性因子的影响 [J].儿科药学杂志, 2019, 25(4): 35-37.

[24] 陈列强, 陈文慧. 连花清瘟颗粒治疗小儿肺炎支原体肺炎 [J].中国卫生标准管理，2020,11（11）：104-106.

[25] 董樑, 夏敬文, 龚益等. 连花清瘟胶囊治疗慢性阻塞性肺 疾病急性加重期的临床疗效和作用机制 [J].中成药, 2014, 36 (5) : 926-929.

[26] 陈波, 姚扬伟, 王海琴．连花清瘟颗粒联合莫西沙星片治 疗老年社区获得性肺炎疗效观察 [J]. 吉林医学, 2013, 34 (1) :79-80.

[27] 吕宏, 欧阳张宁, 王俊等. 连花清瘟胶囊联合左氧氟沙星 治疗肺炎的临床研究 [J]. 现代药物与临床, 2018 , 33(9):2294-2297. 